Original article

\title{
Visceral Leishmaniasis Eradication is a Reality: Data from a Community-based Active Surveillance in Bangladesh
}

\author{
Farhana Ferdousi ${ }^{1} \uparrow$, Mohammad S. Alam ${ }^{2} \uparrow$, Mohammad S. Hossain ${ }^{2}$, Enbo Ma ${ }^{1}$, Makoto Itoh ${ }^{3}$, Dinesh Mondal ${ }^{2}$, \\ Rashidul Haque ${ }^{2}$ and Yukiko Wagatsuma ${ }^{1 *}$ \\ Received 30 September, 2012 Accepted 12 November, 2012 Published online 22 December, 2012
}

\begin{abstract}
More than 20 million people in Bangladesh are considered at risk of developing visceral leishmaniasis (VL). A community-based active surveillance was conducted in eight randomly selected villages in a highly endemic area of Bangladesh from 2006 to 2008. A total of 6,761 individuals living in 1,550 mud-walled houses were included in the active surveillance. Rapid rK39 dipstick tests were conducted throughout the study period to facilitate the case diagnosis. Individuals with previous or current clinical leishmaniasis were identified on the basis of the case definition of the VL elimination program. Untreated cases of suspected VL were referred to the hospital for treatment. Socioeconomic and environmental information including bed net use was also collected. In 2006, the annual incidence of clinical leishmaniasis in the study area was 141.9 cases per 10,000 population, which was significantly increased by the following year owing to community-based active surveillance for case detection and reporting. However, early case detection and early referral for treatment led to a significant decrease in incidence in 2008. This study suggests that community-based active surveillance using a simple diagnostic tool might play a role in achieving the goal of the VL elimination program.
\end{abstract}

Key words: visceral leishmaniasis, active surveillance, rK39 dipstick test, risk factors, Bangladesh

\section{INTRODUCTION}

Visceral leishmaniasis (VL), or kalaazar, is one of the most neglected tropical diseases in the world, affecting the poorest segments of rural populations. In the Indian subcontinent, VL was almost eliminated as a collateral effect of the malaria eradication program during the 1960 s, which was largely based on the widespread use of DDT residual spraying [1]. A resurgence of the disease occurred during the late $1970 \mathrm{~s}$, when large-scale use of DDT was stopped [2]. According to the Directorate General of Health Services in Bangladesh, a total of $70,170 \mathrm{VL}$ cases were reported throughout the country during the previous 10 years (19992010). However, these official figures are thought to grossly underestimate the real situation.

The unique anthroponotic features of VL in the Indian subcontinent, including Phlebotomus argentipes as the only vector, humans as the only reservoir, and a defined geographical area, make the disease a potential candidate for elimination [3]. Knowledge of this possibility led to the signing of a memorandum of understanding in 2005 by Bangladesh, India, and Nepal to eliminate VL by reducing the incidence of the disease to less than one case per 10,000 population by 2015 . Early case detection and treatment together with integrated vector management are the main strategic pillars of the VL elimination program. However, delays in case detection and treatment remain a problem in the control of VL despite some advancement in diagnostics and treatment.

The objective of this study was to provide information regarding the VL burden in endemic communities of Bangladesh in the early phase of the eradication program

\footnotetext{
${ }^{1}$ Department of Clinical Trial and Clinical Epidemiology, Faculty of Medicine, University of Tsukuba, Tennodai 1-1-1, Tsukuba, Ibaraki 3058575, Japan

${ }^{2}$ Parasitology Laboratory, Laboratory Sciences Division, International Centre for Diarrhoeal Disease Research, Bangladesh (icddr,b), Dhaka1212, Bangladesh

${ }^{3}$ Department of Infection and Immunology, Aichi Medical University School of Medicine, Nagakute, Aichi 480-1195, Japan

*Corresponding author:

Department of Clinical Trial and Clinical Epidemiology, Faculty of Medicine, University of Tsukuba, Tennodai 1-1-1, Tsukuba, Ibaraki 3058575, Japan

Phone \& Fax: +81-29-853-3489

E-mail: ywagats@md.tsukuba.ac.jp

$\uparrow$ These authors contributed equally to this work.
} 
and to identify some of the risk factors associated with VL in these areas. The results of this study might help to demonstrate the potential of active surveillance as an important element of the VL elimination program.

\section{MATERIALS AND METHODS}

Mymensingh is the most endemic district for VL in Bangladesh. Every year, more than $60 \%$ of the total VL cases reported in Bangladesh are from this district. The present study was conducted in Trishal, one of the highest VL case-reporting subdistricts of Mymensingh. Trishal consists of 12 unions with an area of $339 \mathrm{~km}^{2}$ and 80,000 households comprising a total population of 372,000 (according to the 2001 census). Two unions of the Trishal subdistrict were chosen for the study because they had the highest incidence of VL according to hospital data for 2003. From these two unions, eight villages were further selected (Fig. 1). The villages were selected by multicluster random sampling. All the inhabitants living in mud-walled houses were included in the study.

At the beginning of the study, individuals from each selected household were invited to participate in the community-based active surveillance starting in August 2006. Children aged less than three years were excluded from the study because VL is both difficult to diagnose in small children and is also still uncommon in this age group. Field research assistants completed a household roster and recorded individuals with VL in the household within the previous one year as per the study case definition stated below. Untreated VL suspected cases were referred to the government hospital for further confirmation and appropriate case management. Information on demographic, socioeconomic, and educational status and mosquito-control measures was also collected.

To facilitate case management, the rapid rK39 dipstick test was performed for the maximum number of individuals available at the beginning of the study (Kalazar Detect ${ }^{\mathbb{R}}$; InBios International, Seattle, WA, USA). According to a recent meta-analysis study, the average sensitivity and specificity of rK39 dipstick test was $92 \%$ and $95 \%$ respectively [4]. Rapid rK39 dipstick tests were performed at one-year intervals for two consecutive years (in 2007 and 2008) for suspected VL cases. A person with fever $\geq 2$ weeks and splenomegaly was considered a "suspected VL case." RK39positive individuals with signs and symptoms of VL were considered as "probable cases of VL" and referred to the nearby government hospital for further confirmation and treatment. RK39-positive persons without any signs or symptoms were considered "asymptomatic VL patients" and advised to contact the health workers working in the villages for study purposes. In the event of any subsequent complaint of VL symptoms, an additional test was performed and probable VL patients were referred to the government hospital. Field research assistants conducted home visits on holidays and in the early morning and late evening on working days. At least one home visit was conducted every month in each village. More frequent home visits, i.e., at

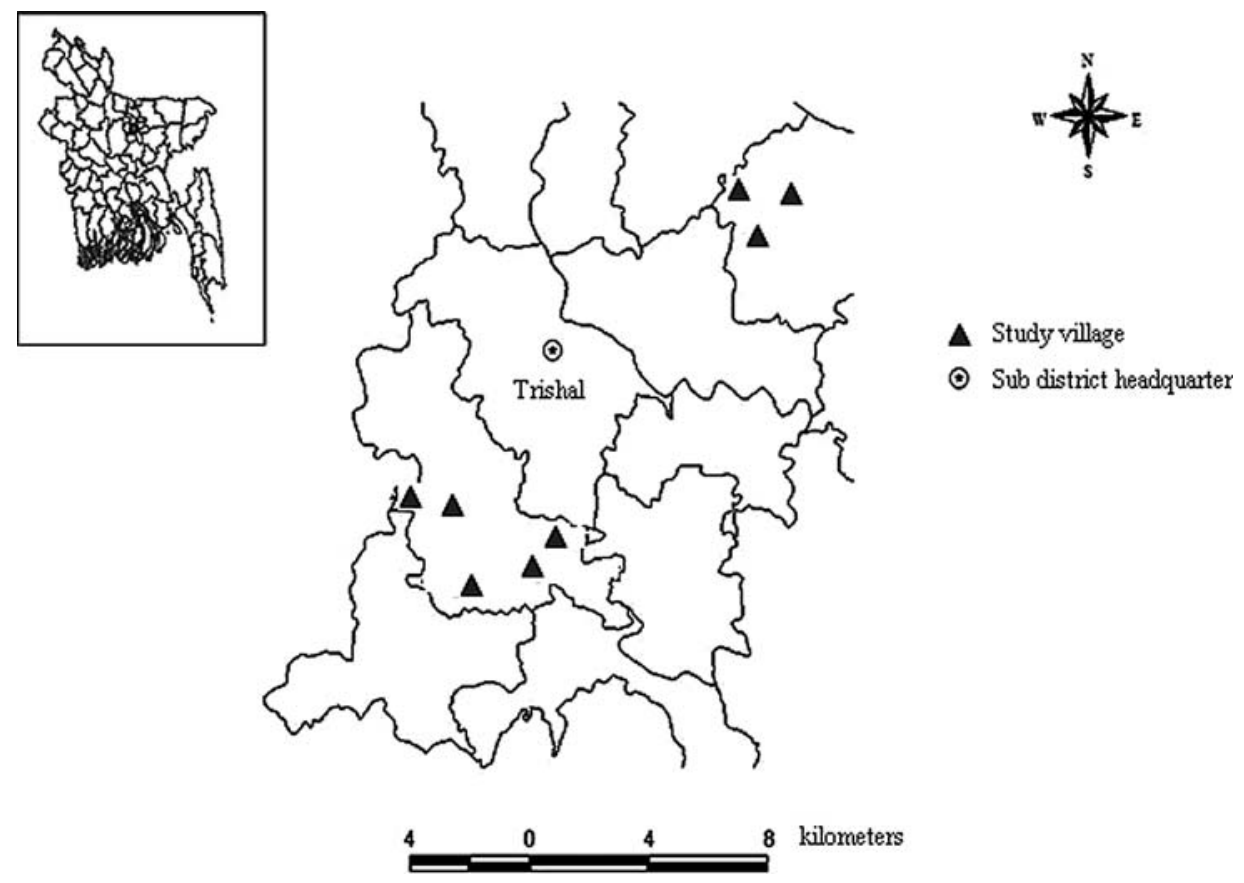

Fig. 1. Study area and villages 
least one home visit every two weeks, were conducted during the summer (April through September). Copies of the treatment record sheets of VL patients were collected from the corresponding government hospital in order to identify patients missing from those referred to the hospital by the field assistants. In such cases, field staff visited the missing patients' houses and assisted them in reporting to the hospital. This longitudinal prospective cohort study was part of a community-based intervention study with a biopesticide (neem oil) [5].

IBM SPSS version 20.0 software was used for the statistical analysis. The general characteristics of the study subjects and households were summarized as the frequency for categorical variables. Age of the study subjects was categorized into three groups, 3-14 years, 15-45 years, and $>45$ years, according to a previous study in Bangladesh which demonstrated high prevalence of VL among younger ( $<45$ years old) people [6]. The incidence of VL was calculated for each year $(2006,2007$, and 2008). Univariate analysis of the association between VL incidence and potential risk factors was conducted using a modified Poisson regression analysis with a robust error variance procedure [7] Cochran-Mantel-Haenszel (CMH) statistics were then conducted to measure the relationships between each pair of VL incidence-related variables identified from univariate analysis. A final multivariate model was constructed to determine the relative associations between VL incidence and each significant variable while adjusted for other covariates. Sex was also included in the final multivariate model according to evidence shown in a previous study [8]. The significance level was set at $p<0.05$ for all tests. The results were expressed as the relative risk (RR) and 95\% confidence interval (CI).

The following study case definitions were used:

Clinical/confirmed leishmaniasis cases (past cases): patient diagnosed with illness characterized by $\geq 2$ weeks of fever and at least one of the following: splenomegaly, skin darkening, and/or weight loss, plus a history of treatment with either sodium stibogluconate or pentamidine with clinical resolution of the symptoms; or with Leishmania amastigotes demonstrated in bone marrow or splenic aspirate or tissue over the past one year and documented in the medical records.

Clinical/confirmed leishmaniasis cases (current cases): patient diagnosed with illness characterized by $\geq 2$ weeks of fever and at least one of the following: splenomegaly, skin darkening, and/or weight loss; or with Leishmania amastigotes demonstrated in relevant aspirate or tissue, and/or a positive rK39 dipstick result.

\section{ETHICAL APPROVAL}

The protocol was approved by the icddr,b Research and Ethical Review Committee. Informed consent was obtained from all adult participants and from a parent or guardian of all participating children.

\section{RESULTS}

A total of 1,550 mud-walled houses accommodated 6,955 inhabitants in the study villages. Household interviews were successfully completed for all mud-walled houses. Active disease surveillance was conducted for 6,761 individuals aged $\geq 3$ years in 2006 . Table 1 shows the individual and household characteristics of the study subjects. Most of the inhabitants $(99.7 \%)$ had been living in the study area for more than three years. About one third $(34.7 \%)$ were children aged less than 15 years. The average number of household members was $4(\mathrm{SD}=2)$ and the average household yearly income was US $\$ 526.0$ (SD = 333.6). More than $70 \%$ of the household heads were illiterate, and only $12.2 \%$ had completed $\geq 5$ years of institutional education. The majority of households $(59.5 \%)$ owned land and at least one domestic animal (72.9\%). Only 23.4\% households used some kind of mosquito-control measures; however, most of them $(74.1 \%)$ were irregular in implementing those measures. Although $92.1 \%$ of the households used bed nets, only $23.8 \%$ of them did so regularly. Table 2 shows the incidence of clinical leishmaniasis from 2006 through 2008. In 2006, 96 individuals were identified as having clinical leishmaniasis. In 2007, the number of reported clinical leishmaniasis cases was substantially increased over that of the previous year $(\mathrm{RR}=1.38 ; 95 \% \mathrm{CI}=$ 1.07-1.79). However, the incidence of clinical leishmaniasis in 2008 was significantly lower than in $2006(\mathrm{RR}=0.19$; $95 \% \mathrm{CI}=0.12-0.32$ ).

Table 3 shows the results of the Poisson regression analyses for variables significantly associated with clinical leishmaniasis. It was found that VL incidence was significantly different among the three age groups (likelihood ratio $\left.\chi^{2}=14.78, \mathrm{df}=2, p=0.001\right)$. The younger age group (3-14 years) was 2.17 times more at risk of developing VL than the older age group of $>45$ years $(\mathrm{RR}=2.17 ; 95 \% \mathrm{CI}=$ $1.39-3.37, p=0.001)$; but the age group of $15-45$ years showed borderline significance compared to the older age group of $>45$ years $(\mathrm{RR}=1.55 ; 95 \% \mathrm{CI}=0.99-2.41, p=$ $0.055)$. Male participants tended to develop VL more frequently $(4.1 \%)$ than female participants $(3.2 \%)$, but the difference was not statistically significant $(\mathrm{RR}=1.26 ; 95 \% \mathrm{CI}$ $=0.98-1.61, p=0.066$ ). People living without electricity in their homes were at a higher risk of developing VL than 
Table 1. Characteristics of the study subjects $(n=6761)$ and households $(n=1550)$

\begin{tabular}{|c|c|c|}
\hline & No. & Percentage \\
\hline \multicolumn{3}{|l|}{ Age, years $(n=6761)$} \\
\hline $3-14$ & 2344 & 34.7 \\
\hline $15-45$ & 3365 & 49.8 \\
\hline$>45$ & 1052 & 15.6 \\
\hline \multicolumn{3}{|l|}{$\operatorname{Sex}(n=6761)$} \\
\hline Male & 3429 & 50.7 \\
\hline Female & 3332 & 49.3 \\
\hline \multicolumn{3}{|l|}{ Education of household head, years $(n=1550)$} \\
\hline 0 & 1129 & 72.9 \\
\hline $1-5$ & 232 & 15.0 \\
\hline$>5$ & 189 & 12.2 \\
\hline Have own land $(\mathrm{n}=1550)$ & 923 & 59.5 \\
\hline Have electricity in the house $(n=1550)$ & 168 & 10.8 \\
\hline Share a bedroom with others $(\mathrm{n}=1550)$ & 1110 & 71.6 \\
\hline Have domestic animals $(n=1550)$ & 1130 & 72.9 \\
\hline Have a cattle shed on the premises $(n=1550)$ & 671 & 43.3 \\
\hline Use mosquito-control measures at night $(\mathrm{n}=1550)$ & 363 & 23.4 \\
\hline Mosquito coil & 131 & 36.1 \\
\hline Smoke (burning straw etc.) & 232 & 63.9 \\
\hline \multicolumn{3}{|l|}{ Frequency of use of mosquito-control measures $(n=363)$} \\
\hline Always & 62 & 17.1 \\
\hline Sometimes & 269 & 74.1 \\
\hline Only in summer & 32 & 8.8 \\
\hline Use bed net at night $(\mathrm{n}=1550)$ & 1428 & 92.1 \\
\hline \multicolumn{3}{|l|}{ Frequency of bed-net use $(n=1428)$} \\
\hline Always & 340 & 23.8 \\
\hline Sometimes & 953 & 66.7 \\
\hline Only in summer & 135 & 9.5 \\
\hline
\end{tabular}

Table 2. Incidence of clinical leishmaniasis in the study area

\begin{tabular}{lrcc}
\hline & No. & $\begin{array}{c}\text { Incidence } \\
\text { (per 10,000) }\end{array}$ & $\begin{array}{c}\text { Relative risk (RR) } \\
(95 \% \text { CI) }\end{array}$ \\
\hline Clinical leishmaniasis in 2006 & 96 & 141.9 & reference \\
Clinical leishmaniasis in 2007 & 133 & 196.7 & $1.38^{*}(1.07-1.79)$ \\
Clinical leishmaniasis in 2008 & 19 & 28.1 & $0.19^{* *}(0.12-0.32)$ \\
\hline
\end{tabular}

${ }^{*} p<0.05, * * p<0.001$

people who did have it $(\mathrm{RR}=3.40 ; 95 \% \mathrm{CI}=1.76-6.59$, $p<0.001)$. Similarly, people who never used mosquitocontrol measures or bed nets while sleeping were more prone to VL than those who had at least some habit of using a bed net or other mosquito-control measure ( $(\mathrm{RR}=1.49$; $95 \% \mathrm{CI}=1.09-2.06, p=0.013$, and $\mathrm{RR}=2.02 ; 95 \% \mathrm{CI}=$ $1.44-2.84, p<0.001$, respectively). The other variables, i.e., education of household head, having own land, having domestic animal, and having cattle shed on the premises, were not found to be significantly associated with clinical leishmaniasis (data not shown).

The younger age group (3-14 years) implemented fewer mosquito-control measures than the older age group $>45$ years $(24.8 \%$ and $27.6 \%$ respectively, $p=0.082)$. Moreover, the male younger age group used fewer mosquito-control measures than the female younger age group $(24.5 \%$ and $25.2 \%$ respectively, $p=0.372)$. On the other hand, use of bed nets was slightly higher among male 
Table 3. Factors related to clinical leishmaniasis

\begin{tabular}{|c|c|c|c|}
\hline & \multirow{2}{*}{$\begin{array}{c}\text { Clinical leishmaniasis } \\
\text { No. of cases }\end{array}$} & \multirow{2}{*}{$\begin{array}{c}\text { Univariate analysis }^{\dagger} \\
\text { RR }(95 \% \mathrm{CI})\end{array}$} & \multirow{2}{*}{$\begin{array}{c}\text { Multivariate analysis } \\
\text { RR }(95 \% \text { CI })\end{array}$} \\
\hline & & & \\
\hline \multicolumn{4}{|l|}{ Age, $y$} \\
\hline $3-14$ & 111 & $2.17(1.39-3.37)^{* *}$ & $2.17(1.39-3.37)^{* *}$ \\
\hline $15-45$ & 114 & $1.55(0.99-2.41)$ & $1.59(1.02-2.47)^{*}$ \\
\hline$>45$ & 23 & reference & reference \\
\hline \multicolumn{4}{|l|}{ Sex } \\
\hline Male & 140 & $1.26(0.98-1.61)$ & $1.26(0.99-1.62)$ \\
\hline Female & 108 & reference & reference \\
\hline \multicolumn{4}{|c|}{ Have electricity in the house } \\
\hline No & 239 & $3.40(1.76-6.59)^{* * *}$ & $2.99(1.56-5.75)^{* *}$ \\
\hline Yes & 9 & reference & reference \\
\hline \multicolumn{4}{|c|}{ Use mosquito-control measures at night } \\
\hline Never & 203 & $1.49(1.09-2.06)^{*}$ & $1.41(1.03-1.92)^{*}$ \\
\hline Always/sometimes & 45 & reference & reference \\
\hline \multicolumn{4}{|l|}{ Use bed net at night } \\
\hline Never & 37 & $2.02(1.44-2.84)^{* * *}$ & $1.96(1.40-2.75)^{* * *}$ \\
\hline Always/sometimes & 211 & reference & reference \\
\hline
\end{tabular}

Univariate Poisson regression analysis

Multivariate Poisson regression analysis adjusted for age, sex, having electricity in the house, use of mosquito-control measures at night, and use of bed nets at night

${ }^{*} p<0.05, * * p<0.01, * * * p<0.001$

than female participants $(92.2 \%$ and $91.9 \%$ respectively, $p=$ $0.695)$. However, these associations were not statistically significant. Nor was a significant association found between using a bed net and implementation of mosquito-control measures $(p=0.068)$. A significantly positive association was found between having electricity in the house and implementing mosquito-control measures $(p<0.001)$ and between having electricity in the house and using a bed net $(p<0.001)$.

Table 3 shows the result of multivariate Poisson regression analysis of the final model adjusted for all the covariates with a $p$ value of $<0.05$ in the univariate analysis. After adjustment, the age group of 15-45 years became statistically significant $(\mathrm{RR}=1.59 ; 95 \% \mathrm{CI}=1.02-2.47, p=$ $0.040)$. The association between having electricity and clinical leishmaniasis was slightly attenuated, but still remained significant $(\mathrm{RR}=2.99 ; 95 \% \mathrm{CI}=1.56-5.75, p=0.001)$. A similar result was found for use of mosquito-control measures $(\mathrm{RR}=1.41 ; 95 \% \mathrm{CI}=1.03-1.92, p=0.031)$ and use of bed net $(\mathrm{RR}=1.96 ; 95 \% \mathrm{CI}=1.40-2.75, p<0.001)$.

\section{DISCUSSION}

The results of this study suggested that VL was underreported in 2006 through the existing passive case detection system. The increased incidence of clinical leishmaniasis in
2007 indicates that community-based active surveillance using a simple diagnostic tool (rK39 dipstick test) can substantially increase the level of case reporting. Early case reporting and referral for treatment could significantly reduce the source of infection within the community, which resulted in the notably decreased incidence of clinical leishmaniasis in 2008. In South Asia, as in Bangladesh, delays in case detection and treatment remain a problem in the control of VL. The median delay from onset of fever to treatment was reported to be about four months [9-11]. Moreover, the number of people exposed to infection or infected without any symptoms has important implications in disease transmission. Therefore, early diagnosis with active surveillance and early treatment are essential not only to cure the VL patient but also to decrease the infection reservoir. The rapid rK39 dipstick test has shown high sensitivity and specificity in detecting VL infections on the Indian subcontinent [12]. The World Health Organization (WHO) currently recommends it as the best available diagnostic tool for VL for use at health facilities in remote areas. Thus, this kind of simple diagnostic tool may improve active surveillance programs by facilitating case management.

Although this study was not designed for comprehensive risk factor analysis, we tried to identify some of the factors associated with clinical leishmaniasis. This study found that the children (3-14 years old) of these communities 
were at a higher risk of developing VL than the older age group of $>45$ years, as in other endemic areas of Bangladesh [6]. It was also found that male cases of VL were more frequent than female cases, although the difference was not statistically significant. A recent study in Nepal found that male participants had a significantly higher risk of developing VL than female participants [8]. Other studies in Bangladesh and India did not find any association between sex and VL incidence [6, 13]. Traditionally, men in the countryside keep the upper part of their body exposed and wear fewer clothes than women, particularly in the summer months, which might make them more vulnerable to bites by the sandfly vector. We also found that the use of some mosquito-control measure, e.g., smoke and mosquito coil, provided protection against VL. However, very few households reported implementing such measures regularly. Moreover, people in the younger age group, especially young males, used fewer mosquito-control measures than people in the older age group, which might also make them more susceptible to the sandfly bite.

Previous epidemiologic studies on the Indian subcontinent found bed nets to be a protective factor against VL [6, 9], which is also supported by our findings. More than $90 \%$ of households in the study villages reported using bed nets at night. This high percentage suggested that bed nets were already widely implemented in Bangladeshi communities. However, only $23.8 \%$ of the households reported using bed nets regularly. Previous studies in this subcontinent region demonstrated that insecticide-treated bed nets were a favorable option for vector management [14, 15]. Therefore, health education programs regarding personal protection measures followed by provision of insecticide-treated bed nets promise to be a highly effective prevention intervention in the endemic community.

$\mathrm{VL}$ is known as a disease of the poorest people on the Indian subcontinent [16]. Although determining the relationship between poverty and VL involves multiple factors [17], poverty has been found to be associated with VL in previous studies conducted in this region [9, 10]. In the present study, having electricity in the household, which may constitute an indicator of better socioeconomic status, was found to reduce the risk of developing VL. Previous studies in Nepal [8,9] and Bangladesh [6] found that ownership of cattle was strongly protective against VL. Another study in India found that illiteracy was associated with VL risk [13]. However, in the present study, neither cattle ownership nor illiteracy of the household head was associated with VL incidence in the univariate analysis and thus was not included in the multivariate analysis.

This study was part of a community-based intervention study with neem oil. Neem oil was not found to be effective in the control of VL; in fact the VL incidence was slightly higher in the intervention area. Probably the result was confounded by other variables such as better socioeconomic condition and higher rate of using mosquito-control measures including bed nets in the control area. Thus, neem intervention was not included in the analysis. In this study, we did not report some of the important factors of recent interest, such as immunogenetic factors, dietary indicators, and nutritional status, which might influence the susceptibility of a host to the development of VL infection [6, 18]. Availability of a highly sensitive and specific rapid diagnostic test (rK39), an increasing number of treatment options, and the unique anthroponotic features of the sandfly vector make VL a potential candidate for elimination. However, massive efforts in community-based active disease surveillance coupled with scaled-up personal protection measures and integrated vector management interventions are required to achieve the goal of the VL elimination program.

\section{ACKNOWLEDGMENTS}

Muhammad Z. Islam and Kazi M. Jamil are gratefully acknowledged for their valuable advice and support for the study. We also thank the field research assistants for their dedicated work in conducting this study. We are grateful to the study participants for their cooperation. We kindly thank Ms. Flaminia Miyamasu for her professional revision of the English text. This study was funded by a research grant for international medical cooperation (kokui-shitei-004) from the Ministry of Health, Labor and Welfare, Japan.

\section{REFERENCES}

1. Alam MS, Wagatsuma Y, Mondal D, Khanum H, Haque R. Relationship between sand-fly fauna and kala-azar endemicity in Bangladesh. Acta Trop 2009; 112: 23-25.

2. Elias M, Rahman AJ, Khan NI. Visceral leishmaniasis and its control in Bangladesh. Bull World Health Organ 1989; 67(1): 43-49.

3. WHO. Regional strategic framework for elimination of kala-azar from the south-east Asia region (2005-2015). SEA-VBC-85 (Rev.1) 2005.

4. Maia Z, Lirio M, Mistro S, Mendes CMC, Mehta SR, Badaro R. Comparative study of rK39 leishmania antigen for serodiagnosis of visceral leishmaniasis: Systematic review with meta-analysis. PLoS Negl Trop Dis 2012; 6(1): e1484.

5. Wagatsuma Y, Alam MS, Fukushige M, Islam MZ, Itoh M, Mondal D, Haque R. Neem extract as a control tool for vector-borne diseases: an example of visceral leishmaniasis in Bangladesh. Biopestic Int 2009; 5(2): 134-140.

6. Bern C, Hightower AW, Chowdhury R, Ali M, Amann J, Wagatsuma Y, Haque R, Kurkjian K, Vaz LE, Begum M, 
Akter T, Cetre-Sossah CB, Ahluwalia IB, Dotson E, Secor WE, Breiman RF, Maguire JH. Risk factors for kala-azar in Bangladesh. Emerg Infect Dis 2005; 11(5): 655-662.

7. Zou G. A modified Poisson regression approach to prospective studies with binary data. Am J Epidemiol 2004; 159(7): 702-706.

8. Rijal S, Uranw S, Chappuis F, Picado A, Khanal B, Paudel IS, Andersen EW, Meheus F, Ostyn B, Das ML, Davies C, Boelaert M. Epidemiology of Leishmania donovani infection in high-transmission foci in Nepal. Trop Med and Int Health 2010; 15(Suppl. 2): 21-28.

9. Bern C, Joshi AB, Jha SN, Das ML, Hightower A, Thakur GD, Bista MB. Factors associated with visceral leishmaniasis in Nepal: bed-net use is strongly protective. Am J Trop Med Hyg 2000; 63(3-4): 184-188.

10. Thakur CP. Socio-economics of visceral leishmaniasis in Bihar (India). Trans R Soc Trop Med Hyg 2000; 94(2): 156-157.

11. Ahluwalia IB, Bern C, Costa C, Akter T, Chowdhury R, Ali M, Alam D, Kenah E, Amann J, Islam M, Wagatsuma Y, Haque R, Breiman RF, Maguire JH. Visceral leishmaniasis: consequences of a neglected disease in a Bangladeshi community. Am J Trop Med Hyg 2003; 69(6): 624-628.

12. Sundar S, Singh RK, Bimal SK, Gidwani K, Mishra A, Maurya R, Singh SK, Manandhar KD, Boelaert M, Rai M. Comparative evaluation of parasitology and serological tests in the diagnosis of visceral leishmaniasis in India: a phase III diagnostic accuracy study. Trop Med Int Health
2007; 12(2): 284-289.

13. Ranjan A, Sur D, Singh VP, Siddique NA, Manna B, Lal CS, Sinha PK, Kishore K, Bhattacharya SK. Risk factors for Indian kala-azar. Am J Trop Med Hyg 2005; 73(1): 74 78.

14. Picado A, Das ML, Kumar V, Kesari S, Dinesh DS, Roy L, Rijal S, Das P, Rowland M, Sundar S, Coosemans M, Boelaert M, Davies CR. Effect of village-wide use of longlasting Insecticidal nets on visceral leishmaniasis vectors in India and Nepal: a cluster randomized trial. PLoS Negl Trop Dis 2010; 4(1): e587.

15. Mondal D, Chowdhury R, Huda MM, Maheswary NP, Akther S, Petzold M, Kumar V, Das ML, Gurung CK, Ghosh D, Kroeger A. Insecticide-treated bed nets in rural Bangladesh: their potential role in the visceral leishmaniasis elimination programme. Trop Med Int Health 2010; 15(11): 1382-1389.

16. Boelaert M, Meheus F, Sanchez A, Singh SP, Vanlerberghe V, Picado A, Meessen B, Sundar S. The poorest of the poor: a poverty appraisal of households affected by visceral leishmaniasis in Bihar, India. Trop Med Int Health 2009; 14(6): 639-644.

17. Alvar J, Yactayo S, Bern C. Leishmaniasis and poverty. Trends Parasitol 2006; 22(12): 552-557.

18. Karplus TM, Jeronimo SM, Chang H, Helms BK, Burns TL, Murray JC, Mitchell AA, Pugh EW, Braz RF, Bezerra FL, Wilson ME. Association between the tumor necrosis factor locus and the clinical outcome of Leishmania chagasi infection. Infect Immun 2002; 70(12): 6919-6925. 